\title{
ACTIVISMO POLÍTICO DE LA SOCIEDAD CIVIL MIGRANTE Y GOBIERNO LOCAL: LA POLÍTICA PÚBLICA MIGRATORIA EN SAN FRANCISCO
}

\author{
POLITICAL ACTIVISM OF THE MIGRANT CIVIL SOCIETY AND LOCAL \\ GOVERNMENT: MIGRATION PUBLIC POLICY IN SAN FRANCISCO
}

\author{
Paola Virginia Suárez Ávila \\ Centro de Investigaciones y Estudios Superiores \\ en Antropología Social CIESAS, México D.F. México/Mexico \\ caraxola@gmail.com
}

Recibido/Received: 29/11/2011

Modificado/Modified: 02/04/2012

Aceptado/Accepted: 09/04/2012

\section{RESUMEN}

El presente artículo parte del análisis de datos históricos y etnográficos que permitan analizar; 1) las interacciones políticas entre sociedad civil y gobiernos local y estatal alrededor del campo de política pública migratoria de la ciudad de San Francisco, California; 2) con base en el estudio de los actores societales y gubernamentales que entran en relación en un campo específico de la acción política migratoria; 3) que expresan proyectos políticos culturales para el desarrollo local de políticas públicas en materia migratoria; 4) los cuales expresan las trayectorias y repertorios de acción de los grupos sociales y políticos que están interrelacionados en el proceso y 5) son estudiadas por medio del análisis de interfaces socio-estatales.

\section{PALABRAS CLAVES}

Desarrollo local, gobierno local, interfaz, migración, activismo político y política pública.

\section{SUMARIO}

1. Introducción. 2. Propuesta teórico-metodológica. 3. El impacto de la aplicación de las leyes migratorias federales en las comunidades de San Francisco. 4. De los movimientos sociales a la política pública: nuevos espacios de participación política de la sociedad civil migrante. 5 . Conclusiones. Bibliografía.

\begin{abstract}
This article stems from the analysis of historical and ethnographic data: (1) political interactions between civil society and local and state governments concerning the field of migration public policy in the city of San Francisco, California; (2) based on the governmental and societal actors that are interrelated in a specific field of immigration policy; (3) that express cultural-political projects for local development of public policy in immigration matters; (4) which express the trajectories and codes of action of the social and political groups which are interrelated in the process, and 5) are studied through the analysis of socio-state interfaces.
\end{abstract}

\section{KEYWORDS}

Local development, local government, interface, migration, political activism and public policy. 


\section{CONTENTS}

1. Introduction. 2. Theoretical-methodological proposal. 3. The impact of the implementation of federal immigration laws on the communities of San Francisco. 4. From social movements to public policy: new spaces of political participation in migrant civil society. 5. Conclusions. References

\section{INTRODUCCIÓN}

Los estudios antropológicos sobre la relación sociedad civil-Estado han resultado una corriente de estudio novedosa que renueva el análisis de la diversidad de interacciones entre Estado y sociedad civil en campos específicos de la política. La presente investigación se inserta en los estudios de política migratoria local en Estados Unidos enfocados en el estudio de políticas públicas y el activismo político en materia de derechos de los inmigrantes de la ciudad y condado de San Francisco, California. Los conceptos teórico metodológicos que guían la investigación parten de la hipótesis de que los gobiernos locales y la sociedad civil, agrupada en organizaciones políticas de San Francisco, han buscado nuevas rutas de discusión para solucionar los problemas en materia migratoria por medio del activismo político para hacer frente al poder casi absoluto del gobierno federal de Estados Unidos en materia migratoria.

El proceso de política pública en materia migratoria de la ciudad de San Francisco es analizado por medio de interrelaciones entre sociedad civil-gobierno local y estatal. Estas interrelaciones son reconocidas en el estudio como interfaces, comprendidos también como espacios de intercambio entre la sociedad y el estado para la resolución de problemas de una comunidad sociopolítica impactada por la migración internacional. Dado que el gobierno local de San Francisco y los grupos por los derechos de los inmigrantes son actores heterogéneos que expresan la diversidad del activismo político de la Bahía de San Francisco en una coyuntura política especial, el debate de la Reforma Migratoria en la administración del presidente Obama, es importante acotar la investigación en la arena política sobre la discusión de los derechos de los inmigrantes para conocer las dinámicas de coacción entre estos dos actores, sociedad civil y gobierno -local y estatal.

El artículo analiza un estudio de caso de política pública en materia migratoria, deconstruido en los espacios de interrelación ente un grupo de la sociedad civil, Grupo por una alternativa de legislación migratoria, promovido por la Coalición por los Derechos de los Inmigrantes de la Bahía de San Francisco (BAIRC, por sus siglas en inglés) y el gobierno local de San Francisco. Es interés particular analizar las interacciones entre Estado y sociedad civil para conocer cuáles son las interfaces en las que están relacionados, los dispositivos que integran dichas interfaces y el sistema migratorio federal que actualmente funciona y limita la acción política del gobierno local de San Francisco para gestionar derechos y servicios de los inmigrantes en el ámbito local.

Esta perspectiva relacional analiza este segmento de la realidad como un proceso de políticas públicas que configura el escenario contemporáneo en el que identificamos actores sociales, societales, gobierno y agencias de rendición de cuentas que están en disputa y en comunicación constante para el reconocimiento de derechos de los migrantes en una esfera local. 


\section{PROPUESTA TEÓRICO - METODOLÓGICA}

El presente artículo aborda el fenómeno migratorio como objeto de política pública, esto es como área de intervención socio-estatal. La intervención socio-estatal es entendida, según la define Norman Long, como un concepto deconstruido que implica analizar un proceso en movimiento, socialmente construido, negociado, experiencial y creador de significados, no simplemente la ejecución de un plan de acción ya especificado con resultados de comportamientos ya esperados. La propuesta teórico metodológica parte del análisis de interfaces propuesto por Norman Long, quien explica que "con el fin de examinar estas interrelaciones es útil trabajar con el concepto de "interfaz social", el cual explora las maneras en las que discrepancias de interés social, interpretación cultural, conocimiento y poder son mediadas y perpetuadas o transformadas en puntos críticos de eslabonamiento o confrontación. Estas interfaces necesitan ser identificadas etnográficamente, no conjeturadas con base en categorías predeterminadas" como se menciona en Long (2007:105-109).

El concepto de interfaz está expresado como aquellos espacios donde existe intercambio y conflicto entre actores diversos que se relacionan de manera intencional. Un interfaz es una entidad organizada de intencionalidades y relaciones entrelazadas; aparece como un lugar para el conflicto, la incompatibilidad y la negociación y donde se confrontan paradigmas culturales. Está constituido por el poder, entendido como luchas estratégicas y significados y compuesta por discursos múltiples; implica también una realidad donde los procesos de conocimiento son centrales y puede ser el espacio para la intervención planeada en los mundos de vida de los actores sociales individuales y colectivos Long (2007:105-109).

El espacio social está definido cómo aquél divido en campos o esferas donde los sujetos están relacionados por una serie de bienes, y en los que la distribución se traduce en luchas por tales bienes. Está constituido de tal forma que los agentes se distribuyen en él en función de su posición según principios de diferenciación, como el capital económico y el capital cultural (Bourdieu, 1990 y Isunza y Hevia: 2006).

Un campo social define lo qué está en juego y los intereses específicos, su estructura es un estado de relación de fuerzas entre los agentes o las instituciones que intervienen en la lucha. La diversidad de actores que participan en la lucha tiene que ver con una cantidad de intereses fundamentales comunes (Ver: Servicio). Para comprender los interfaces es necesario establecer una línea de los contornos y contenidos de formas sociales diversas, explicando su génesis y trazando sus implicaciones para la acción estratégica y modos de conciencia. Por ello, es importante comprender los proyectos político-culturales de los actores sociales y estatales para analizar las relaciones o interfaces socio-estatales que se dan en el campo de acción de la política migratoria en San Francisco (Long, 2007:105-109).

Aplicando el modelo teórico de interfaces, encuentro la riqueza para discutir las diferentes interrelaciones que existen entre organizaciones civiles y políticas por los derechos de los migrantes y el gobierno local de San Francisco en la discusión de la Reforma Migratoria de Estados Unidos. Básicamente porque las interfaces ayudan a explicar la arena política en la que interaccionan diferentes actores sociales y estatales en la búsqueda de soluciones a la política migratoria y el fortalecimiento de la política pública de la ciudad y condado de San Francisco en materia de Migración y para el desarrollo local de las comunidades de migrantes. En la actualidad, con el cambio de administración del presidente George Bush a la nueva administración del presidente Barack Obama en enero de 2009, ha comenzado una nueva discusión sobre el tema de la Reforma Migratoria en diversos ámbitos sociales, políticos, laborales, culturales y religiosos y es un común consenso entre la sociedad 
civil, los inmigrantes y el sistema de gobierno de Estados Unidos que el sistema migratorio en Estados Unidos está en franca crisis y es necesario solucionarlo en los años siguientes.

El trabajo de campo realizado en la ciudad y condado de San Francisco, California, durante agosto de 2008 junio de 2010 tuvo como objetivo principal conocer el proceso de políticas públicas en materia migratoria de la ciudad de San Francisco y conocer cómo discuten organizaciones y gobierno local para lograr una alternativa de legislación en materia migratoria que discuta la propuesta de Reforma Migratoria Comprensiva desarrollada en la Casa Blanca de Washington, DC. En la agenda política de diversas organizaciones de activistas latinos, de defensa de los inmigrantes y de derechos humanos en Estados Unidos existe la urgencia de promover la discusión y reforma de las actuales leyes de inmigración. De todo este universo de actores societales, he elegido trabajar con la Coalición por los Derechos de los Inmigrantes de la Bahía de San Francisco (Bay Area Immigrant Rights Coalition, BAIRC) porque han conjuntado los esfuerzos de diversas organizaciones asiáticas, latinas y mexicano -americanas de defensa de los inmigrantes y de derechos humanos.

\section{EL IMPACTO DE LA APLICACIÓN DE LAS LEYES MIGRATORIAS FEDERALES EN LAS COMUNIDADES DE SAN FRANCISCO}

En los Estados Unidos, el Departamento de Seguridad Interna (Department of Homeland Security, DHS) se ha vuelto el centro de la política de inmigración desde los atentados a las torres gemelas el 11 de septiembre de 2001. La responsabilidad de la inmigración se comparte en tres oficinas de departamento: el Servicio de Inmigración y Control de Aduanas de Estados Unidos (ICE) y Aduanas y Protección Fronteriza (CBP) comparten la responsabilidad de aplicar la ley, mientras que Servicios de Ciudadanía e Inmigración (CIS) es responsable de inmigración y naturalización (Varsanyi, 2010). Según la página oficial del ICE, es la mayor entidad investigadora del Departamento de Seguridad Nacional. Fue creado en 2003, dos años después de los atentados del 11 de septiembre de 2001 para combinar la acción de las dependencias que trabajaban bajo las órdenes de los anteriores Servicio de Inmigración y Naturalización y el Servicio de Aduanas de Estados Unidos (Ver: Servicio).

El 12 de febrero de 2009 en la reunión mensual de la Comisión de Derechos Humanos de San Francisco (Human Rights Coalition, HRC, por sus siglas en Inglés), Christopher Punongbayan de la Comisión de Derechos de los Inmigrantes (Immigration Rights Coalition, IRC, por sus siglas en inglés) y Director de la organización Asian Law Caucus, invitó a la Comisión a ser co-participante de la Audiencia sobre los efectos de las políticas migratorias del ICE y las actividades relacionadas en la ciudad y condado de San Francisco (Ver: Gobierno). Las comisiones de Derechos Humanos (HRC) y Derechos de los Inmigrantes (IRC) son agencias del gobierno local de San Francisco que tienen como objetivo conocer la aplicación de leyes del gobierno local y federal en materia de derechos humanos y de los inmigrantes en el condado y ciudad de San Francisco.

El comisionado de la IRC, Punongbayan, explicó que durante el verano de 2008 las comunidades inmigrantes del Mission District y Tenderloin habían sido objeto de violaciones de sus derechos humanos registrándose redadas, despidos masivos y detenciones ilegales. Después de la discusión, fue aprobada una resolución por la Comisión de Derechos Humanos (HRC) de San Francisco para convocar a una Audiencia 
Pública donde los individuos y organizaciones de inmigrantes expusieran los impactos de la aplicación de leyes de Inmigración y la acción del ICE en San Francisco. El 13 de abril de 2009, las Comisiones de Derechos de los Inmigrantes y de Derechos Humanos de San Francisco convocaron a los miembros de todas las comunidades de inmigrantes de San Francisco a la Audiencia titulada "The Impact of Immigration Enforcement on San Francisco Communities", la cual se realizó en la sala de la junta de supervisores del Palacio Municipal de San Francisco. La Audiencia tuvo como objetivo conocer cómo las familias, los individuos y los negocios de San Francisco han sido impactados por la presencia del Servicio de Inmigración y Control de Aduanas de Estados Unidos (ICE).

La invitación fue expresa para que los inmigrantes de distintas comunidades emitieran sus puntos de vista y compartieran su experiencia sobre el impacto de la aplicación de las leyes federales de inmigración en sus vidas, incluyendo el acceso a la salud, la educación y el empleo. Realizaron una especial invitación a los miembros de la comunidad que habían sido sujetos de violaciones a sus derechos civiles y humanos para que compartieran su experiencia con los comisionados. A las seis de la tarde de ese día en el Palacio Municipal asistieron a la convocatoria más de 200 personas de diversos grupos migratorios quienes rindieron testimonio durante más de tres horas. Se proveyó traducción en español, cantonés (idioma de la República de China) y tagalog (idioma oficial de Filipinas). En la sala, organizadores, inmigrantes, activistas y agentes públicos esperaban con ansia el suceso. Algunos organizadores empezaron a circular volantes e información, así como calcomanías para pegarse en la solapa del saco, las que decían, por ejemplo: "Hablar español no es un delito", "Justice for immigration rights", etc.

En el evento estuvieron presentes los miembros de la Comisión de Derechos de los Inmigrantes y los supervisores David Chiu, presidente de la Junta de Supervisores y Supervisor del Distrito 3, David Campos del Distrito 9 y John Avalos del Distrito 11. En el Distrito 3 habita una gran parte de la comunidad migrante asiática e integra en sus territorios el Chinatown; en los distritos 9 y 11 habita la mayor parte de los latinos e inmigrantes latinoamericanos de San Francisco y ahí se ubica el Mission District. Los primeros oradores fueron los tres supervisores mencionados anteriormente. David Chiu expresó que la historia de San Francisco ha sido construida por los inmigrantes; John Avalos dijo que esta era una oportunidad de la comunidad y que también era un momento para replantear qué es lo que significa que San Francisco sea una Ciudad Santuario; por último, David Campos explicó que la historia de los inmigrantes ha sido una historia de miedo, aunque los avances en materia migratoria de la ciudad de San Francisco han ayudado a que las políticas locales transformen la vida de los migrantes en un sentido positivo pero es necesario hacer más transformaciones y modificar la ley de la Ciudad Santuario de San Francisco.

La ley de Ciudad Santuario fue aprobada por la Junta de Supervisores de San Francisco en 1989. Dicha ley dice que "Ningún departamento, agencia, comisión, oficial o empleado de la Ciudad y Condado de San Francisco podrá usar fondos ni recursos Municipales para asistir con la aplicación y cumplimiento de las leyes federales de inmigración ni para recaudar o difundir información respecto al estatus migratorio de individuos en la Ciudad y Condado de San Francisco, a menos que la mencionada asistencia sea requerida por estatutos federales o estatales, normativa, o decisión judicial" (Ver: Gobierno). Actualmente, la ley funciona como un elemento discursivo y ampara a la ciudad y condado contra la colaboración en programas del sistema federal migratorio como 287(g) y Comunidades Seguras. 
Después de la apertura del evento encabezada por los Supervisores Chiu, Campos y Avalos de los Distritos 3, 9 y 11 respectivamente, un académico de la Universidad de California en Berkeley, ofreció una perspectiva analítica sobre el impacto de las leyes federales y locales en la comunidad de inmigrantes de San Francisco. Goodwin Liu, profesor de Derecho y asociado del decano de la Universidad de California en Berkeley, criticó las declaraciones del Presidente Obama que dicen que en los momentos de recesión económica después de la crisis de 2008, el gobierno debía darle prioridad al empleo. El profesor explicó que los inmigrantes se enfrentan en este momento a un problema de segregación cuando el gobierno ha dicho que trabajará para ayudar a los desempleados. Los inmigrantes no son desempleados, son inmigrantes. No cuentan en este tipo de censos. Anotó que la administración de Obama ha iniciado con buenas y malas noticias. Las buenas son la transición de gobierno, las malas que el gobierno se está enfocando en los desempleados, que no son los inmigrantes ilegales, si no los estadounidenses que son censados. Como propuesta dijo que la nueva administración debe poner énfasis en las leyes de trabajo y dejar en un segundo plano la generación de empleos. Para que se organicen los trabajadores ilegales es necesario modificar las leyes laborales ya que son una importante fuerza laboral; por lo menos en California, que son más del $10 \%$ del total de la fuerza laboral. Goodwin Liu mencionó que el trabajo de las Comisiones de Derechos Humanos y de Derechos de los Inmigrantes, así como otras agencias gubernamentales de San Francisco es hacer una diferencia en la formulación de leyes laborales junto con las comunidades y organizaciones de inmigrantes.

Después de las participaciones del profesor Goodwin Liu, comenzaron los testimonios de cada uno de los individuos y organizaciones que quisieron participar en la Audiencia. El tiempo de exposición de cada uno fue de 3 minutos sin posibilidad de extensión. Aproximadamente 30 individuos y organizaciones dieron testimonio sobre las problemáticas de los inmigrantes en San Francisco y las violaciones a los derechos humanos y de los inmigrantes. La diversidad de los testimonios muestra las problemáticas que tiene el gobierno local de la ciudad para enfrentar el problema de acceso a derechos y a la justicia del sector migrante. En resumen, las demandas fueron las siguientes:

- Detener la revisión de inmigrantes en el Aeropuerto de San Francisco basada en el perfil racial.

- Defender el matrimonio gay para que aquellos inmigrantes que tienen una pareja residente puedan obtener los derechos que otorga el matrimonio legal.

- Defender el presupuesto a las asociaciones de inmigrantes que pelean por sus derechos.

- Detener las redadas del ICE en la ciudad de San Francisco, en especial en el Mission District, y en los centros donde prestan servicios los jornaleros.

- Reformar y revisar la Ley de Ciudad Santuario de San Francisco.

- Defender una justa reforma migratoria a nivel local, estatal y federal.

- Defender el acceso a la salud de los inmigrantes ilegales y trabajadores de baja renta.

- Otorgar identificaciones y licencias de manejo a los inmigrantes.

- Proteger a los trabajadores inmigrantes contra los despidos masivos.

- Proteger a los inmigrantes en desalojo de vivienda.

- Eliminar la discriminación por lenguaje.

- Defender el derecho de residir legalmente en California. 
Frente a los testimonios no hubo resoluciones; el testimonio de la gente sirvió a la Comisión para analizar la problemática local en materia de derechos humanos y de los inmigrantes y convocar a una segunda reunión de trabajo para realizar una serie de recomendaciones al gobierno local de San Francisco.

\section{Activismo político local en materia migratoria}

Este relato etnográfico es un ejemplo de activismo político local en materia migratoria y un interfaz donde participa sociedad, sociedad civil y gobierno local de San Francisco, California. La discusión sobre los efectos de la política migratoria del ICE en las comunidades de inmigrantes de San Francisco convocada por las Comisiones de Derechos Humanos y Derechos de los Inmigrantes de San Francisco es una de las muchas acciones políticas destinadas a la resolución del conflicto que existe entre el sistema federal migratorio y el gobierno local de San Francisco en el campo de la política migratoria.

La ciudad de San Francisco, así como otras entidades políticas locales de Estados Unidos han actuado, desde hace ya algunos años por la búsqueda de la transformación del sistema migratorio federal, para manifestar la necesidad de gobiernos locales y estatales de participar activamente en la discusión y toma de decisiones en política pública migratoria en espacios locales y estatales. Este fenómeno conocido como activismo político local y estatal en materia migratoria ha emergido recientemente como resultado de los límites legales que tienen el gobierno federal en materia de migración para resolver los problemas de los gobiernos locales frente al reconocimiento de derechos y otorgamiento de servicios públicos y sociales para los inmigrantes.

Los antecedentes del emergente fenómeno del activismo político en la primera década del siglo XXI están relacionados con el histórico "pleno poder" del gobierno federal sobre asuntos de inmigración y la relegación de los gobiernos locales y estatales en el dominio de la hechura de política pública en materia de migración (Varsanyi, 2010). El pleno poder del gobierno federal en materia migratoria se refiere a la histórica absorción de todos los poderes (federal, estatal y local) en agencias federales que guían y vigilan la política migratoria de Estados Unidos incluyendo dos áreas, la política nacional que busca la integración o exclusión de los inmigrantes en las comunidades políticas de Estados Unidos y, en la política exterior, que limita el libre tránsito e intercambio del flujo humano, así como sus fronteras del exterior al estado-nación.

La reciente emergencia del activismo local y estatal en política migratoria levanta interesantes cuestiones que miran no solo a la constitucionalidad de dichas políticas pero también al cambio y apropiado rol de los gobiernos locales en la formación de política migratoria. Frente al poder pleno del gobierno federal en materia migratoria, los gobiernos locales plantean nuevos retos y soluciones para el conflicto de las comunidades locales con las comunidades de inmigrantes. El activismo local y estatal en política migratoria ha tenido un auge en los años recientes. Comenzando con la Proposición 187 de California, también conocida como "Salva nuestro estado", la mayoría de los votantes de California aprobaron dicha propuesta de ley el 8 de noviembre de 1994. La proposición buscaba prevenir que los inmigrantes ilegales en el estado de California accedieran a ciertos servicios públicos financiados por el gobierno estatal, incluyendo la salud pública y la educación (desde K12 hasta la Universidad); así como permitir que todas las agencias locales de aplicación de la ley del estado cooperaran con las agencias federales para la aplicación de la ley federal de inmigración. 
Desde mitades de la década de los noventa con la aprobación del Tratado de Libre Comercio de América del Norte (TLCAN) hasta nuestros días en Estados Unidos han sido aprobadas diversas políticas migratorias. Algunas políticas han intensificado la militarización en la frontera México-Estados Unidos, la criminalización de los inmigrantes en el sistema estadounidense, la reducción de los derechos sociales y culturales. Otras han procurado el acceso a servicios de salud, educación y vivienda de los inmigrantes. Aun así se han generado pocas propuestas, políticas y/o leyes para cambiar el estatus legal de los trabajadores indocumentados e inmigrantes que arriban y/o residen en las diversas ciudades de Estados Unidos.

Este corpus de políticas, que varían en la dirección, pueden ser interpretadas como política migratoria local, algo que desde el siglo XIX en los Estados Unidos había sido imposible constitucionalmente y es aparece a finales del siglo XX como un fenómeno emergente de la política pública local migratoria en dicho país. Estas nuevas políticas migratorias vuelven borrosa y cruzan la línea entre las políticas de integración migratoria política pública- y las políticas de control migratorio -técnicamente considerado política exterior en los Estados Unidos (Varsanyi, 2010). El tema de la Reforma Migratoria ha sido intensamente debatido por más de una década en los Estados Unidos por distintos grupos de la sociedad civil nacional e internacional y por los gobiernos locales y el gobierno federal de Estados Unidos; así como por organismos y gobiernos internacionales.

En 2001, después de los atentados del 11 de septiembre, la estrategia política del gobierno federal de Estados Unidos y sus agencias transformaron las políticas de migración y derechos de los inmigrantes para detener el "terrorismo". El Acta Patriótica aprobada ese mismo año por el Congreso de los Estados Unidos dio poderes a las agencias federales para investigar y castigar a todos los ciudadanos e inmigrantes sospechosos de crímenes en contra de la nación estadounidense y su población. Dos años después, creó el Servicio de Inmigración y Control de Aduanas (US Immigration and Customs Enforcement, ICE) para el cumplimiento de las leyes de inmigración y aduanas y proteger a Estados Unidos de ataques terroristas. El ICE se concentra en los inmigrantes ilegales, y las personas, el dinero y los materiales que prestan apoyo al terrorismo y otras actividades criminales.

Con base en estas nuevas políticas federales de migración, en San Francisco se han reportado conflictos entre la policía y la comunidad de inmigrantes, en especial los inmigrantes de origen latinoamericano que habitan en el Distrito de la Misión (Mission District). Los documentos de trabajo de la Comisión de Derechos de los Inmigrantes de San Francisco, una agencia de rendición de cuentas, reporta que han sido sujetos a violaciones como deportaciones, detenciones, redadas y despidos masivos las comunidades de inmigrantes de San Francisco, en especial, en el Distrito de la Misión donde se concentra la población migrante de Latinoamérica. Desde la Alcaldía y el Departamento de Policía de San Francisco, hay reportes de que el ICE ha buscado la cooperación con las agencias gubernamentales del gobierno local y la policía local para la detención de inmigrantes sospechosos por medio del Programa Comunidades Seguras; programa que viola el Acta de Ley Santuario que niega cualquier participación de las autoridades locales con las federales para detener inmigrantes.

La ciudad y condado de San Francisco tiene sus propias leyes que amparan a los inmigrantes en esta región sociopolítica. El Acta de Ciudad y Condado Santuario ampara a los inmigrantes frente a las acciones del gobierno federal y sus agencias para la detención e investigación de sospechosos; explícita que no es posible que la policía y las agencias gubernamentales locales cooperen con el ICE y el sistema migratorio federal. El gobierno 
local de San Francisco también ha avanzado en la cuestión de la legislación migratoria en el ámbito local. Ha establecido agencias especiales que son las instancias encargadas de gestionar las políticas públicas dirigidas a los inmigrantes: La Comisión de los Derechos Humanos (HRC), la Comisión de los Derechos de los Inmigrantes (IRC) y la Oficina de Asuntos de Migración y Compromiso Cívico. Dichos organismos son las instancias del gobierno local que interactúan con los inmigrantes y han estado al frente de un nuevo debate sobre Reforma Migratoria para hacer un análisis sobre el impacto y la forma en que la ciudad y condado de San Francisco pueden participar en el debate nacional.

\section{DE LOS MOVIMIENTOS SOCIALES A LA POLÍTICA PÚBLICA: NUEVOS ESPACIOS DE PARTICIPACIÓN POLÍTICA DE LA SOCIEDAD CIVIL MIGRANTE}

El concepto de sociedad civil migrante se refiere a las organizaciones representativas de base; específicamente, en cuatro ámbitos de acción colectiva: 1) organizaciones de base cuyos miembros y dirigentes son inmigrantes; 2) medios de comunicación de y para inmigrantes; 3) ONG conducidos por migrantes y 4) espacios públicos autónomos de migrantes (Fox, 2005:42). La sociedad civil migrante de San Francisco ha fomentado la construcción de un movimiento social y político importante a favor de los derechos de los inmigrantes y trabajadores de baja renta. Organizaciones de inmigrantes asiáticos, latinos y de la izquierda de Estados Unidos han comprendido la importancia de colaborar juntos para enfrentar los embates de las nuevas regulaciones del gobierno y agencias federales que afectan las libertades de las comunidades inmigrantes de San Francisco. Libertades como el libre tránsito, la libertad de asociación y otras garantías que otorga la Constitución de los Estados Unidos han sido discutidas frente a la legislación migratoria.

El movimiento social y de organización social y política multiétnica en San Francisco es comparable con otros movimientos que se dan en áreas metropolitanas de California y Estados Unidos: Los Ángeles, Chicago, Nueva York, Washington, Houston y Phoenix. En cada una de estas áreas hay una diversidad social, cultural y política única y distinta que brinda nuevos caminos de discusión para que la sociedad civil migrante conozca mejores vías para solucionar por caminos democráticos el problema de la migración y el acceso a derechos de los inmigrantes a nivel local y federal.

La ciudad de San Francisco tiene una historia interesante de activismo y organización civil y política que parte de la sociedad civil y del gobierno. Es particular de la ciudad la gran cantidad de organizaciones (actualmente están registradas 7.093 organizaciones) de índole diversa que se ocupan de cooperar y ayudar a ciertos grupos minoritarios y/o comunidades marginadas como la comunidad lésbica, gay, bisexual y trans (transgénero, travestis y transexuales), LGBT, la latina y los vagabundos (homeless) por medio de otorgar servicios que son concedidos con capital privado y del gobierno estatal y local. Dichas organizaciones sirven para obtener servicios como salud, educación y vivienda, así como iniciar luchas por medio de la participación ciudadana y buscar la comunicación con los departamentos, agencias y otras instancias gubernamentales locales, federales y nacionales para promover discusiones locales que busquen una mejoría de grupos minoritarios (Silverman et al. 2009:11).

Muchas de las organizaciones brindan servicios a los trabajadores indocumentados, inmigrantes y residentes de la ciudad que no tienen el estatus de ciudadano y no pueden acceder a servicios de salud, educación, vivienda y mejorar las condiciones laborales. 


\section{La Misión de San Francisco}

En el distrito 9 de San Francisco, conocido como La Misión, habita un gran número de la población latina de la ciudad y también en él hay una gran cantidad de organizaciones (aproximadamente 50 organizaciones) que apoyan y brindan servicios a la población inmigrante, trabajadores de bajos recursos y residentes de origen latino y asiático (Silverman et al. 2009:11). La población latina que vive en la ciudad de San Francisco es mayoritariamente mexicana y centroamericana, la gran parte de ellos se dedican principalmente a trabajar en restaurantes, hoteles, jardinería, limpieza, construcción, textiles, cargadores en los puertos y otros servicios (Passel, 2005:29).

Esta población latina reside en la Misión de San Francisco. Este espacio geográfico es conocido como barrio mexicano o latino por la comunidad de San Francisco. La Misión funciona como un barrio mexicano con sus tiendas de la esquina, sus fruterías y verdulerías, sus panaderías, sus cantinas y sus peluquerías; con tiendas de música ranchera, iglesias católicas, murales en las calles con imágenes de la Virgen de Guadalupe, muy lejos del sueño americano. Cuando uno camina por sus calles, el mayor número de transeúntes es de origen latinoamericano y el idioma español es predominante; se ven grupos de hombres jóvenes de rasgos indígenas; a las mujeres se les ve en los restaurantes sirviendo, preparando comida o caminando en la calle con niños, también caminan algunas prostitutas de origen latino; se escucha la música norteña, los corridos, la salsa y el reggaetón; los anuncios de los comercios están en español y pocos aparecen en inglés; circulan los periódicos locales en español, se escucha la radio y la tele local en español. El barrio se observa pobre con relación a su pasado de bonanza encerrado en las calles.

Las organizaciones y los líderes latinos de la Misión trabajan conjuntamente con la población para mejorar los estándares de vida, que no se reducen simplemente a promover el acceso a la ciudadanía en Estados Unidos, sino a la posibilidad de una comunidad deprimida social, política, cultural y económicamente para mejorar las condiciones de vida a un nivel comunitario y no individual, como sería el simple acceso al derecho de la ciudadanía. Las formas de acción y organización de la comunidad latina en la Misión son muchas; pero todas, tienen en común el interés de que dicha comunidad acceda a mejores servicios y obtenga mayores ingresos económicos que les permitan tener una mayor movilidad social, económica y política a nivel local, regional y nacional.

En el movimiento social que se viene gestando en la Misión actúan diversos grupos societales, conformando estrategias para el desarrollo comunitario para promover la discusión y encontrar alternativas de acción por vías democráticas y la consolidación de proyectos socio-políticos y económicos que ayuden a la integración económica de los inmigrantes y sus familias. La Coalición multiétnica elegida para el análisis ha sido Coalición por los Derechos de los Inmigrantes de la Bahía de San Francisco conformada por organizaciones filipinas, latinoamericanas y latinas, una organización no gubernamental y un comité de estadounidenses a favor de los derechos de los inmigrantes. Las organizaciones que han participado en la discusión más activamente son: American Friends Service Committe (AFSC), Filipinos for Affirmative Action, Bay Area Immigrant Rights Coalition (BAIRC), La Raza's Day Laborer Project y David Bacon . Una coalición, un comité, dos organizaciones étnicas, una de filipinos y otra de latinos, y un activista independiente. Elegí este grupo por tres criterios de análisis principalmente: 1) la participación de líderes latinos y asiático-americanos; 2) la comunicación con otras organizaciones étnicas y a favor de los derechos de los inmigrantes y trabajadores de bajos recursos; 3) la relevancia del tema para la comunidad migrante latinoamericana de la región. Cada uno de los criterios de análisis 
enuncia algunas de las características de la participación cívica, política, social y cultural de la sociedad civil migrante y latina en el área de la Bahía de San Francisco.

El primer criterio, indica la importancia de la participación de la sociedad civil migrante y los líderes mujeres y hombres, la cual ha sido primordial en los años recientes y coloca a dicho grupo en una nueva condición histórica frente a la sociedad y al sistema político estadounidense, adquiriendo una voz y un espacio en la esfera pública, siendo que su acción transforma su condición en la escena pública y política a nivel local, nacional y binacional.

El segundo apunta a la importancia de que dicho grupo interactúe y se comunique con otros grupos sociales y minorías étnicas para hacer posible la creación de estrategias, como la formación de Coaliciones, lazos y redes de solidaridad y apoyo para la construcción de espacios de discusión y participación política de la sociedad civil migrante latinoamericana y latina.

El tercer punto indica las temáticas y problemáticas que discute la sociedad civil migrante y latina en la región en este momento, lo que implica los intereses y las prácticas políticas, civiles y culturales que los hace reunirse, participar y actuar frente a situaciones precisas y problemas de dicho grupo.

\section{Grupo por una alternativa de legislación migratoria}

La Coalición por los Derechos de los Inmigrantes (BAIRC) es una coalición fundada en 2001. Los antecedentes de la organización tienen que ver con importantes movimientos y acción social de otras coaliciones y grupos de trabajo para discutir la Reforma Migratoria y los efectos de las actuales leyes migratorias de Estados Unidos. La Coalición lanzó una convocatoria en marzo de 2009 para formar un grupo de discusión sobre la Reforma Migratoria y conformar el Grupo por una alternativa de legislación migratoria. Una propuesta alternativa de reforma migratoria en San Francisco implica para este grupo la posibilidad de crear nuevas redes entre la sociedad civil y los gobiernos a nivel local, estatal y federal para realizar una propuesta de ley que signifique más a los inmigrantes que a las necesidades del sistema de migración en Estados Unidos y la creación de movimientos sociales.

El grupo tiene la capacidad de comunicarse con las comunidades migrantes de San Francisco por medio de la participación cotidiana y la cooperación para resolver conflictos a nivel local. La información que recopilan las distintas organizaciones y activistas del grupo implica un esfuerzo de reflexión de las propias comunidades sobre su participación en la vida social, económica, política y cultural y contiene fuentes de información diversas de las comunidades migrantes sobre sucesos y eventos que los han marcado frente en situaciones concretas de violación de derechos humanos y de los inmigrantes. El grupo conoce la importancia de que el trabajo debe partir de campañas educativas y de difusión, así como generar la opinión pública entre las comunidades de inmigrantes y la sociedad civil. Hacen énfasis en la ampliación del movimiento, por ello, han creado la "Campaña por la Dignidad". La campaña clarifica la posición del grupo en puntos clave para hacer que la sociedad civil y agencias gubernamentales interesadas discutan las posibilidades y estrategias para mejorar la calidad de vida de los inmigrantes por medio de la reformación del sistema migratorio de los Estados Unidos desde la experiencia local.

La "Campaña por la Dignidad" pretenden disminuir los problemas de injusticia, desigualdad y violación de derechos de los inmigrantes a nivel local y nacional, reducir el poder de las agencias gubernamentales para que los gobiernos locales puedan tener poder de decisión sobre las acciones en contra de jóvenes e inmigrantes apresados por delitos distintos a los que explícita el sistema migratorio; proteger los derechos de los trabajadores 
e indocumentados; así como luchar por el derecho de asociación y organización de los trabajadores ilegales para que aboguen en uniones, sindicatos, organizaciones y otro tipo de asociaciones y eliminar el sistema E-verify y otros sistemas que reporta a empleadores inconsistencias en los datos de los trabajadores como el número de seguridad social.

\section{Los nuevos espacios de discusión pública de los gobiernos local y estatal}

El análisis de la política migratoria en San Francisco abre las puertas para el conocimiento de una sociedad política que ha tenido una intensa y compleja historia de activismo social donde las corrientes políticas de izquierda y del bienestar social han influido para la resolución de conflictos de los grupos minoritarios, incluidos los inmigrantes según Richard DeLeon (1988). Según este mismo autor, la ciudad de San Francisco es la capital del progresismo en Estados Unidos porque ha crecido como una ciudad que no limita la dependencia política y económica al sector privado y su capital, creando un liderazgo progresista que planea la autonomía local, expande la esfera pública, politiza y democratiza los procesos de planeación, restringe el uso del comercio en el espacio urbano y busca limitar el poder del sector privado frente a los intereses de las comunidades.

La ciudad de San Francisco ha tenido importantes movimientos y activismo social que promueve la discusión de proyectos políticos locales con un gran número de actores sociales en una esfera pública. El concepto de la esfera pública surge entre el dominio de la autoridad pública o del Estado, de un lado, y el dominio privado de la sociedad civil y de la familia, del otro, como una esfera de lo público integrada por individuos privados que discuten la regulación de la sociedad civil y la administración del Estado (Habermas, 1987).

Siendo que es una ciudad progresista, busca la comunicación de distintos agentes políticos y sociales para la construcción de decisiones y políticas públicas, creando así una esfera pública específica y particular que se genera en San Francisco y que impacta a las comunidades del área de la Bahía de San Francisco como las ciudades de Oakland, San José, Berkeley, Richmond, entre otras. La Comisión Little Hoover de California ha informado en un sumario ejecutivo que las políticas públicas en materia migratoria entre el estado de California y el gobierno federal son inconsistentes porque algunas leyes federales excluyen a algunos inmigrantes para recibir beneficios de la seguridad social, siendo que California (como gobierno) ofrece a ellos pagos suplementarios que compensen los servicios no otorgados por el gobierno federal.

La Comisión Little Hoover, formalmente conocida como Milton Marks "Little Hoover" Comisión sobre la organización gubernamental y económica del Estado de California, es una agencia independiente del Estado creada en 1962 y establecida en Sacramento. La misión de la comisión es investigar operaciones del gobierno estatal por medio de reportes, recomendaciones y propuestas legislativas para promover la eficiencia, economía y eficacia de los servicios. Por estatuto, la Comisión está balanceada por una junta bipartidista compuesta por cinco miembros ciudadanos propuestos por el Gobernador, cuatro por la legislatura, dos por los senadores y dos por la Asamblea (Little, 2010).

Según el sumario anteriormente citado, la única política pública que es consistente en el sistema es la de Salud donde todos los inmigrantes son excluidos de los servicios de Medi-Cal, sistema de salud pública brindado por el gobierno de California. Sin embargo, existen otros servicios comunitarios donde los inmigrantes pueden acudir y recibir los servicios auspiciados por organizaciones y gobiernos locales y estatal (Habermas, 1987). Asimismo, menciona el documento que algunas de las leyes en materia migratoria castigan o impiden el acceso de servicios por igual a inmigrantes legales o ilegales, convirtiéndolos 
en un grupo vulnerable. Además, existe la queja de que la aplicación de leyes del sistema federal hace que los oficiales estén ocupados de su aplicación en vez de proteger la seguridad pública; que el INS gasta muchos millones en la protección de la frontera México-Estados Unidos; e irónicamente, el Internal Revenue Service (IRS, por sus siglas en inglés) aplica impuestos a los inmigrantes ilegales (Little, 2010).

Según el informe, las políticas públicas del sistema federal enfocadas en los estatus migratorios a menudo frustran la habilidad de las comunidades y gobiernos locales para cumplir las prioridades y las necesidades de los más de 2 millones de inmigrantes ilegales y de los 8 millones de migrantes que viven en California. Explican que un problema fundamental es cómo son definidos los inmigrantes por las políticas públicas y tratados en los programas públicos, es el resultado de una compleja serie de leyes, normas y regulaciones que se han desarrollado en los últimos años (Little, 2010). Los problemas mencionados por la Comisión Little Hoover están presentes en las demandas públicas de las asociaciones por los derechos de los inmigrantes de San Francisco.

En San Francisco hay un activismo político importante que negocia la agenda pública sobre migración y que parte de diversos actores societales y gubernamentales para solucionar problemas que existen en el sistema federal migratorio y que afecta a las comunidades de migrantes. Hay más de 80.000 unidades gubernamentales en los Estados Unidos y cada una puede considerarse un sistema político, entre ellas el gobierno local de San Francisco. El sistema político estadounidense se basa en la idea del federalismo y en el reconocimiento de la autonomía en toma de decisiones, procuración de la justicia y establecimiento de leyes de los estados y gobiernos que la conforman. Además, los municipios (condados) tienen sus propios gobiernos y agencias para hacer valer las leyes locales y federales.

La dinámica del federalismo en Estados Unidos hace que los modelos políticos sean distintos; en las relaciones intergubernamentales están expresadas sus variedades siendo que un caso específico puede ser la ciudad y condado de San Francisco que cuenta con organismos gubernamentales específicos para hacer valer la justicia y el poder de los ciudadanos en una esfera local frente a los gobiernos estatales y el federal. Sin embargo, dentro de la legislación migratoria existe un poder pleno del gobierno federal que explicita que ninguna ley local o estatal puede legislar localmente. Lo que ha accedido el poder federal representado por la Suprema Corte es aceptar cualquier regulación en materia migratoria que sea armoniosa con las leyes federales.

\section{CONCLUSIONES}

Estados Unidos contemporáneo ofrece un amplio campo de políticas públicas locales para el análisis comparativo. Como un campo de estudio, la hechura de política migratoria en un nivel local es importante por distintas razones. Primero, políticas locales y estatales pueden afectar los resultados de las políticas de migración a nivel nacional -su eficacia y la uniformidad de sus efectos. Segundo, las medidas locales y estatales pueden ir más lejos que las leyes federales, hacia un camino o hacia otro, y esto es substancialmente importante. Tercero, la colisión de las políticas gubernamentales locales y estatales con el sistema judicial federal crea nuevas tensiones, generalmente resultas por las cortes que bloquean o atenúan la implementación de dichas políticas.

Finalmente, las políticas públicas locales pueden tener impactos significativos en la vida diaria. Lo que pasa en los estados y las localidades pueden afectar las condiciones de empleo 
de los inmigrantes, la vivienda, la educación, la salud y la posibilidad de los hijos de los inmigrantes más de lo que pueden las leyes federales y las políticas. El debate de la Reforma Migratoria aun tiene muchos días por delante, así como la discusión de la ley de Arizona y de otras polémicas políticas públicas sobre derechos de inmigrantes en Estados Unidos. Sin embargo, considero importante mencionar que el activismo de inmigrantes y gobierno local de San Francisco y estatal de California han ayudado a que se renueve la discusión y que se encuentren nuevas vías de discusión que mejoren la calidad de vida de los inmigrantes en espacios locales y estatales.

La Reforma Migratoria no es un tema nuevo en la sociedad estadounidense, desde hace más de tres décadas, los migrantes y las agencias de gobierno -federales, estatales y localeshan planteado proyectos político-culturales, que limitan la discusión a puntos específicos de la agenda pública, haciendo visible los espacios de discusión y negociación entre los actores, así como aquellos espacios donde no ha sido posible la comunicación entre ellos, generando incertidumbre e imposibilidades dentro del campo de la política migratoria.

Las interfaces muestran los espacios de interacción de la sociedad civil y el Estado; en este caso, también muestran los espacios donde no se han generado esas interrelaciones y que desde la sociedad civil y el gobierno local pugnan por nuevos espacios de comunicación. En el campo de la política migratoria, después de los atentados del 11 de septiembre de 2001 a las Torres Gemelas en Nueva York, la discusión sobre la Reforma Migratoria se ha convertido en un punto clave de discusión la generación de nuevos espacios de interacción entre agencias gubernamentales, agencias de rendición de cuentas y sociedad civil para responder a las nuevas políticas públicas que han situado en un nuevo proceso a la política pública migratoria a Estados Unidos.

El gobierno local de San Francisco, por medio de agencias de rendición de cuentas, ha establecido una agenda pública que pretende recuperar las voces de muchos individuos y organizaciones que narran, relatan, demandan y promueven el acceso de derechos de los inmigrantes en la sociedad política de San Francisco. El Grupo por una alternativa de legislación migratoria ha generado también la construcción de una agenda pública que sea reconocida por el gobierno local y agencias públicas de Estados Unidos para la consecución de una Reforma Migratoria que parta de una propuesta distinta a la que abogan en Washington bajo el nombre de Reforma Migratoria Comprensiva.

Las propuestas y proyectos socio-políticos que parten de la sociedad civil y de los gobiernos local y estatal están expandiendo la agenda pública propuesta por el gobierno federal en materia migratoria. La apertura de nuevos espacios de discusión en la esfera pública, así como la promoción de políticas públicas locales buscan transformar o limitar el poder pleno del gobierno federal para incluir demandas de las comunidades de inmigrantes y de la sociedad civil migrante en espacios locales.

\section{BIBLIOGRAFÍA}

BLOEMRAAD, I. y KARTHICK RAMAKRISHNA, S. (eds.) (2008), Civic hopes and political realities. Immigrants, community organization and political engagement. Russell Sage Foundation.

BOURDIEU, P. (1990), "Algunas propiedades de los campos", en Sociología y Cultura. México: CONACULTA.

DELEON, R. E. (1988), The progressive urban regime: ethnic coalitions in San Francisco. San Francisco: Public Research Institute, San Francisco State University

FOX J. (2005), Mapping Migrant Civil Society. Santa Cruz: University of California Press. 
Gobierno de San Francisco, "Ciudad y condado refugio" en: [versión electrónica] http://www.sfgsa. org/Modules/ShowDocument.aspx?documentid=1821 (revisado el 05 de julio de 2010).

HABERMAS, J. (1987), Teoría de la acción comunicativa, Tomo II, Crítica de la razón funcionalista. Madrid: Taurus.

Human Rights Commission, "Human Rights Commission. Minutes of February 12, 2009 Meeting" en: [versión electrónica] http://www.sf-hrc.org/Modules/ShowDocument.aspx?documentid =184 (revisado el 15 de julio de 2010)

ISUNZA VERA, E. y HEVIA DE LA JARA, F. (2006), "Relaciones sociedad civil-Estado en México. Un ensayo de interpretación" en Cuadernos para la democratización, núm. 4, CIESAS-UV. México.

LAGUERRE, M. (1999), Minoritized space. An inquiry into the spatial order of things. Berkeley: University of California Press.

LITTLE HOOVER COMMISSION (2010), Executive Sumía, en versión electrónica http://www.lhc. ca.gov/studies/166/execsum166.pdf revisado el 12 de octubre de 2010.

LONG, N. (2007), Sociología del desarrollo: una perspectiva centrada en el actor. México: CIESASColegio de San Luis.

MILKMAN, R. (2000), Organizing immigrants: the Challenge for unions in contemporary California. Ithaca, NY. Cornell University Press.

NGAI, M. M. (2004), Impossible Subjects. Illegal aliens and the making of the modern America. Princeton: Princeton University Press.

PASSEL, J. (2005), Background briefing prepared for task force on Immigration and America's future. Washington, DC.: Pew Hispanic Center. p. 29

PULIDO, L. (2006), Black, brown, yellow and left. Radical activism in Los Angeles. Los Angeles: University of California Press.

QUAN, K. et. al. (2001), Union organizing in California: Challenges and opportunities. Berkeley: University of California Berkeley Press.

Servicio de Inmigración y Control de Aduanas de Estados Unidos, http://www.ice.gov/espanol/about /index.htm.

SILVERMAN, C., et.al (2009), San Francisco’s Nonprofit Sector. San Francisco: University of San Francisco.

VARSANYI M, W. (ed.) (2010), Taking local control. Immigration policy activism in US cities and states. Stanford, CA: Stanford University Press.

\section{Breve currículo:}

\section{Paola Virginia Suárez Ávila}

Candidata a Doctora y Maestra en Antropología Social por el Centro de Investigaciones y Estudios Superiores en Antropología Social (CIESAS) y Licenciada en Historia por la Universidad Nacional Autónoma de México. Realizó una estancia de Investigación Doctoral en el Instituto de Estudios Gubernamentales de la Universidad de California, Berkeley (2008-2011). Ha sido profesora del Colegio de Historia de la UNAM (2005-2008). Becaria CONACyT en el Programa de Postgrado en Antropología Social del CIESAS, DF (2005-2011). Sus líneas de investigación son: Migración, Políticas Públicas, Frontera México/Estados Unidos, Estudios Culturales y Activismo Político de Migrantes. Ha realizado trabajo en la región Tijuana/San Diego y San Francisco, California. Ha participado en eventos académicos y cursos especializados en universidades y centros de investigación de México, Estados Unidos, España, Brasil, Francia y Ecuador. 